\title{
METRIC FLOWS IN SPACE FORMS OF NONPOSITIVE CURVATURE
}

\author{
ARA BASMAJIAN AND GERARD WALSCHAP
}

(Communicated by Christopher Croke)

\begin{abstract}
We characterize those space forms of nonpositive curvature that admit one-dimensional Riemannian foliations. The hyperbolic ones are essentially the trivial line bundles over the flat ones. In particular, any such space admits a flat metric.
\end{abstract}

Let $M$ denote a complete Riemannian manifold. A (nonsingular) foliation $\mathscr{F}$ on $M$ is said to be Riemannian or metric if the leaves remain locally at constant distance from each other. When the leaves are one-dimensional, $\mathscr{F}$ is often referred to as a flow. On generic manifolds, this rarely occurs because of strong restrictions on the curvature. The simply connected space forms of constant curvature $\kappa \leq 0$, on the other hand, admit an abundance of such foliations. When the ambient space is no longer simply connected, some restrictions apply: on compact flat manifolds, for example, any Riemannian foliation is totally geodesic, and vice-versa [Wa]. The situation for compact hyperbolic manifolds is radically different, in that they do not admit foliations of either type [W], [Wa]. A natural question therefore is to determine which space forms of nonpositive curvature can be foliated in such a way. The purpose of this note is to establish the following

Theorem. Let $M^{n}$ be an $n$-dimensional space of constant curvature $\kappa \leq 0, \mathscr{F}$ a metric flow on $M$.

(1) If $\kappa=0$, then $M$ is isometric to $\mathbb{R} \times_{\Gamma} \mathbb{R}^{n-1}$, where $\Gamma=\pi_{1}(M)$ acts diagonally by rigid motions.

(2) If $\kappa<0$, then $M$ is diffeomorphic to $\mathbb{R} \times\left(\mathbb{R} \times_{\Gamma} \mathbb{R}^{n-2}\right)$, with $\Gamma$ as in (1). In particular, $M$ admits a flat metric.

The result applies in particular to space forms that admit a totally geodesic foliation by hypersurfaces, since in this case, the orthogonal complement to the leaves is a Riemannian flow. Moreover, both statements in the above theorem have converses: for example, if $M$ is diffeomorphic to $\mathbb{R} \times\left(\mathbb{R} \times \Gamma \mathbb{R}^{n-2}\right)$ with $\Gamma$ as above, then there exists a hyperbolic metric on $M$ and a flow $\mathscr{F}$ which is Riemannian with respect to that metric.

Received by the editors March 17, 1994.

1991 Mathematics Subject Classification. Primary 53C20, 30F40.

Key words and phrases. Flows, hyperbolic space.

Supported in part by a grant from the National Science Foundation.

(c)1995 American Mathematical Society 
It should be noted that the result does not generalize to nonconstant negative curvature, as can be seen from the following

Example. Let $S$ denote any compact surface with genus $>1$. Then $S \times \mathbb{R}^{2}$ does not admit a flat metric. It does, however, admit a negatively curved metric with a Riemannian flow: let $N$ be the warped product $\mathbb{R} \times_{e^{t}} S$, where $S$ is endowed with a hyperbolic metric (see e.g. [BO] for the definition and properties of warped products). Define $f: N \rightarrow \mathbb{R}$ by $f(t, p):=e^{t}$, and let $M$ denote the warped product $N \times_{f} \mathbb{R} . M$ is diffeomorphic to $S \times \mathbb{R}^{2}$, has negative curvature, and the hypersurfaces $N \times\{t\}$ are totally geodesic in $M$.

Observe also that if one replaces exp by $\cosh$, then $N$ in the above example becomes hyperbolic, with a codimension two totally geodesic foliation (and a Riemannian foliation by hypersurfaces). This shows that the result does not generalize to higher-dimensional foliations.

A key ingredient in the proof is the following

Theorem (Gromoll-Grove [GG1]). Let $\mathscr{F}$ denote a metric flow on a space form of constant curvature. Then $\mathscr{F}$ is flat or homogeneous.

Here, flat means that the distribution orthogonal to $\mathscr{F}$ is integrable (and thus generates a foliation by totally geodesic hypersurfaces), whereas homogeneous means that the lift of $\mathscr{F}$ to the universal cover $\tilde{M}$ of $M$ is spanned by a Killing field on $\tilde{M}$.

The first statement in our theorem now follows easily: indeed, if $M$ is flat, then the Gromoll-Grove result implies that the lifted foliation is always generated by a Killing field $Z$ (which is parallel if $\mathscr{F}$ is flat). Observe that the fundamental group, acting by covering transformations, must preserve the leaves. Now, $Z$ always has at least one geodesic integral curve $c$, and for $g \in \pi_{1}(M)$, $g \circ c$ is again a line, which must be parallel to $c$ since leaves are equidistant. It follows that the linear parts of the covering transformations share a common eigenvector or, equivalently, that the holonomy group has an invariant onedimensional subspace, giving rise to a decomposition $\mathbb{R}^{n}=\mathbb{R} \times \mathbb{R}^{n-1}$. Clearly, the fundamental group acts diagonally on the product, thus establishing (1). Conversely, if $\Gamma$ acts diagonally on $\mathbb{R} \times \mathbb{R}^{n-1}$ by Euclidean isometries, then the homogeneous fibration of $\mathbb{R}^{n}$ by lines parallel to the first coordinate axis is invariant under $\Gamma$, thus inducing a foliation of the same type on the Riemannian quotient $\mathbb{R}^{n} / \Gamma$.

Before turning to the second statement of the theorem, we introduce some terminology. Hyperbolic space $\mathbb{H}^{n}$ is the simply connected complete riemannian manifold of constant sectional curvature negative one. $\mathbb{H}^{n}$ has a compactification which can be realized as the closed unit ball in $\mathbb{R}^{n}$, with the interior of the ball endowed with the Poincare metric. The boundary of this ball is called the sphere at infinity and the action of a discrete subgroup $\Gamma$ of orientationpreserving isometries of $\mathbb{H}^{n}$ extends as conformal mappings to this sphere. The action of $\Gamma$ on the boundary breaks up into the disjoint union of the limit set of $\Gamma$ and the regular (discontinuity) set of $\Gamma$. The group $\Gamma$ is said to be elementary if its limit set contains at most two points. The nontrivial orientation-preserving isometries of $\mathbb{H}^{n}$ are of three types:

(1) hyperbolic; having no fixed points in $\mathbb{H}^{n}$ and two fixed points on the boundary sphere, 
(2) parabolic; having no fixed points in $\mathbb{H}^{n}$ and exactly one fixed point on the boundary sphere,

(3) elliptic; having a fixed point in $\mathbb{H}^{n}$.

The axis of a hyperbolic element $g$ is the unique geodesic in $\mathbb{H}^{n}$ joining the fixed points of $g$. It is necessarily invariant under $g$. In a nonelementary group, there exist two hyperbolic elements having disjoint axes and disjoint fixed point sets. The reader is referred to Beardon [Be] and Maskit [M] for more on the basics. We caution the reader that in this paper hyperbolic isometries include ones that are not pure dilations.

Every hyperbolic manifold (complete orientable Riemannian manifold of constant curvature negative one) is of the form $\mathbb{H}^{n} / \Gamma$, where $\Gamma$ is a torsion-free (no elliptic elements) discrete subgroup of the orientation-preserving isometries of $\mathbb{H}^{n}$.

Now, if the hyperbolic manifold $M^{n}=\mathbb{H}^{n} / \Gamma$ admits a metric flow, then by the Gromoll-Grove result, that foliation is flat (so that its orthogonal complement is a foliation by totally geodesic hypersurfaces), or homogeneous.

\section{THE FLAT CASE}

In the presence of a totally geodesic foliation, using a fact first cited by Zeghib $[Z], \Gamma$ is an elementary group. Furthermore, since $\Gamma$ is the fundamental group of a hyperbolic manifold, it is torsion-free with one or two limit points.

Now, if $\Gamma$ has two limit points, then it is a cyclic group generated by a hyperbolic element whose fixed points constitute the limit set, and hence it is easy to see that $M$ is diffeomorphic to $S^{1} \times \mathbb{R}^{n-1}$ and (2) follows (with, in this case, $\Gamma$ acting trivially on the last factor).

The other possibility is that $\Gamma$ has one limit point, and hence is conjugate to a Euclidean group action. For the remainder of this proof we assume that $\Gamma$ has been normalized so that its limit point is infinity (equivalently that it is a Euclidean group). We claim in this case that the action of $\Gamma$ on the boundary $\mathbb{R}^{n-1}$ of $\mathbb{H}^{n}$ has a totally geodesic codimension one $\Gamma$-invariant foliation. To see this, we need to show that the totally geodesic codimension-one foliation lifted to $\mathbb{H}^{n}$ is by vertical hyperplanes, for then their boundaries are totally geodesic (and $\Gamma$-invariant) in $\mathbb{R}^{n-1}$.

Since a purely elliptic discrete group of Möbius transformations is finite (see [Wat]), the group $\Gamma$ must contain at least one parabolic element $g$, which, up to renormalization, is a glide rotation in the boundary $\mathbb{R}^{n-1}$ with Euclidean axis $A x(g)$. Now, consider the hyperbolic 2-plane $P$ in $\mathbb{H}^{n}$ determined by $A x(g)$. Note that $P$ is $\langle g\rangle$-invariant, $g$ acts as a pure translation on $P$, and that the foliation of $\mathbb{H}^{n}$ induces a totally geodesic $\langle g\rangle$-invariant foliation of $P$. If no leaf of the foliation of $\mathbb{H}^{n}$ contains $P$, then the induced foliation on $P$ is by geodesics and it is not hard to see that any such foliation of $P$ consists entirely of vertical geodesics. Hence, in our situation, if no leaf of the foliation of $\mathbb{H}^{n}$ contains $P$, we can conclude that any leaf passing through $P$ is vertical. Since these leaves foliate all of $\mathbb{H}^{n}$, we are done.

Next, suppose there is a leaf $L$ containing $P$. Then the boundary of $L$ determines a Euclidean hyperplane $Q$ in $\mathbb{R}^{n-1}$ containing $A x(g)$. Now, consider any line $B$ equidistant from $A x(g)$ which is contained in the Euclidean 2-plane perpendicular to $Q$ and containing $A x(g)$. Then clearly $g\left(g^{2}\right.$, if $g$ 
switches components of $\left.\mathbb{H}^{n}-L\right)$ keeps $B$ invariant. The same argument as before for the hyperbolic 2-plane with boundary $A x(g)$ applies and we deduce that either all the leaves in the foliation are vertical, or there is a leaf $L^{\prime}$ of the foliation that contains the hyperbolic 2-plane $P^{\prime}$. We can rule out the first possibility since those leaves would intersect $L$. Thus the boundary at infinity of $L^{\prime}$ determines a Euclidean hyperplane parallel to $L$, and since all such planes fill $\mathbb{R}^{n-1}$, we are finished. Setting $N=\mathbb{R}^{n-1} / \Gamma$, we conclude, since $\Gamma$ preserves (in the upper half-space model) the height of any translated point in $\mathbb{H}^{n}$, that $M$ is $N \times \mathbb{R}$, where $N$ has the desired properties. (2) now follows by applying (1) to the foliated flat manifold $N$.

\section{THE HOMOGENEOUS CASE}

It remains to deal with the case when $\mathscr{F}$ is homogeneous. Let $Z$ denote a nowhere zero Killing field on $\mathbb{H}^{n}$ generating the lifted foliation, and denote by $\left\{\phi_{t}\right\}$ the flow of $Z$. It is straightforward to check that $Z$ is elliptic, hyperbolic, or parabolic, in the sense that for all $t \neq 0, \phi_{t}$ is of that type. Moreover, $Z$ cannot be elliptic-since it is nowhere zero-and all the flow elements share the same fixed points on the boundary. We first show that $\Gamma$ must be elementary: if not, then it contains two hyperbolic elements with disjoint axes and fixed points. It follows that $Z$ cannot be hyperbolic, for otherwise, at least one of the hyperbolic elements $g \in \Gamma$ would have axis $c$ distinct from the axis $\gamma$ of $Z$, and $g \circ \gamma$ would be a second axis for $Z$ since $g$ preserves $\mathscr{F}$. Thus, $\mathrm{Z}$ is not hyperbolic. The one-parameter group $\left\{\phi_{t}\right\}$ of $Z$ then has a unique limit point, and must therefore move at least one of the axes away from itself, say, $\phi_{t}(c(\infty)) \neq c(\infty)$ and $\phi_{t}(c(-\infty)) \neq c(-\infty)$ for all $t \neq 0$. This in turn implies that the Jacobi field $J:=Z \circ c$ has unbounded norm as $t \rightarrow \pm \infty$. Now, if $g$ translates $c$ by $\alpha$, then, since it also preserves $\mathscr{F},\langle J, \dot{c}\rangle /|J|$, which measures the nonoriented angle between $J$ and $c$, must be a periodic function of $t$. This forces the linear function $\langle J, \dot{c}\rangle$ to be constant. If this constant were nonzero, then $|J|$ would be periodic, and $J$ is then everywhere tangent to $c$ since the component orthogonal to $c$ has strictly convex norm if not identically zero. Thus, in our situation, $J \perp \dot{c}$ everywhere. Then $c$ is a horizontal geodesic, and $J$ is a 'holonomy' Jacobi field (see e.g. [GG2]) which is therefore uniquely determined by its value at any one point. Since $g_{*} J$ is again a holonomy field along $t \mapsto c(t+\alpha)$, there exists a constant $\beta$ such that $|J|(t)=\left|g_{*} J\right|(t)=\beta|J|(t+\alpha)$. In particular, if $t_{0}$ is a critical point of $|J|$, then so is $t_{0}+\alpha$. This is clearly impossible, since $|J|$ is strictly convex, unbounded at both ends, and hence assumes a unique minimum. Thus, as claimed earlier, $\Gamma$ must be elementary.

To conclude the proof of the theorem, recall that by the above and the argument for the flat case, $M$ is either $S^{1} \times \mathbb{R}^{n-1}$ or $\mathbb{R} \times N$, where $N$ is flat, and $\Gamma=\pi_{1}(M)$ is, after normalization, a Euclidean group with limit set $\{\infty\}$. In the latter case, $Z$ cannot be hyperbolic, since any nontrivial $g \in \Gamma$, having only one fixed point, would generate a second axis for $Z$. Thus, $Z$ is parabolic. We claim that the limit point $x$ of $Z$ is $\infty$. To see this, let $p$ be an arbitrary point of hyperbolic space, and $H$ the horosphere at $\infty$ through $p$. Choose a nontrivial $g \in \Gamma$, and consider $p_{n}:=g^{n}(p) \rightarrow \infty$. Since $g$ preserves both $H$ and $\mathscr{F}, Z_{p_{n}}$ makes a constant angle with $H$. But $Z_{p_{n}}$ is also tangent to the 
horosphere $H_{n}$ at $x$ through $p_{n}$, and the angle between $H_{n}$ and $H$ goes to 0 as $n \rightarrow \infty$. Thus, $Z_{p_{n}}$ must be tangent to $H$ for all $n$, and since $p$ was arbitrary, $Z$ is tangent to every horosphere at $\infty$, so that $x=\infty$. Since these horospheres cover $N, \mathscr{F}$ restricts to a flow on $N$ which is Riemannian with respect to the flat metric. The statement then follows from (1) in the theorem.

The converse to (2) easily follows from the converse to (1): the homogeneous fibration constructed there extends in a natural way to a $\Gamma$-invariant homogeneous fibration of $\mathbb{H}^{n}$.

As a final remark, it is interesting to note that many of the above arguments carry over to manifolds with curvature bounded above by a negative constant: for instance, if such a manifold admits a homogeneous flow, then its fundamental group is elementary. The proof of the Gromoll-Grove theorem, however, makes extensive use of constant curvature; cf. also [GG2].

\section{REFERENCES}

[Be] A. Beardon, The geometry of discrete groups, Springer-Verlag, New York, 1983.

[BO] R. Bishop and B. O'Neill, Manifolds of negative curvature, Trans. Amer. Math. Soc. 145 (1969), 1-49.

[GG1] D. Gromoll and K. Grove, One-dimensional metric foliations in constant curvature spaces, Differential Geometry and Complex Analysis (I. Chavel and H. Farkas, eds.), Springer-Verlag, New York, 1985, pp. 165-168.

[GG2] The low-dimensional metric foliations of Euclidean spheres, J. Differential Geom. 28 (1988), 143-156.

[M] B. Maskit, Kleinian groups, Springer-Verlag, New York, 1988.

[W] P. Walczak, Dynamics of the geodesic flow of a foliation, Ergodic Theory Dynamical Systems 8 (1988), 637-650.

[Wa] G. Walschap, Foliations of symmetric spaces, Amer. J. Math. 115 (1993), 1189-1195.

[Wat] P.L. Waterman, Purely elliptic Möbius groups, Holomorphic Functions and Moduli II (D. Drasin, C.J. Earle, F.W. Gehring, I. Kra, and A. Marden, eds.), Springer-Verlag, New York, 1988, pp. 173-178.

[Z] A. Zeghib, Laminations et hypersurfaces géodésiques des variétés hyperboliques, Ann. Sci. École. Norm. Sup. (4) 24 (1991), 171-188.

Department of Mathematics, University of Oklahoma, Norman, Oklahoma 73019

E-mail address, A. Basmajian: ara@yankee .math.uoknor.edu

E-mail address, G. Walschap: gwalschap@math.uoknor.edu 